\title{
Preisach modeling of hysteresis for a pseudoelastic Cu-Zn-Al single crystal
}

\author{
Jordi Ortín \\ Departament d'Estructura i Constituents de la Materia, Facultat de Física, Universitat de Barcelona, \\ Diagonal 647, 08028 Barcelona, Catalonia, Spain
}

(Received 3 June 1991; accepted for publication 22 October 1991)

\begin{abstract}
Stress-strain trajectories associated with pseudoelastic behavior of a Cu-19.4 Zn-13.1 Al (at. \%) single crystal at room temperature have been determined experimentally. For a constant cross-head speed the trajectories and the associated hysteresis behavior are perfectly reproducible; the trajectories exhibit memory properties, dependent only on the values of return points, where transformation direction is reverted. An adapted version of the Preisach model for hysteresis has been implemented to predict the observed trajectories, using a set of experimental first-order reversal curves as input data. Explicit formulas have been derived giving all trajectories in terms of this data set, with no adjustable parameters.

Comparison between experimental and calculated trajectories shows a much better agreement for descending than for ascending paths, an indication of a dissymmetry between the dissipation mechanisms operative in forward and reverse directions of martensitic transformation.
\end{abstract}

\section{INTRODUCTION}

The thermoelastic martensitic transition undergone by a number of alloy systems has received considerable attention in the last 15 years, since it is responsible for the striking thermomechanical properties of pseudoelasticity. and shape memory. ${ }^{1}$ Particular attention has been devoted to $\mathrm{Ni}-\mathrm{Ti}$ near the equiatomic composition, ${ }^{2}$ and to copperbased alloys such as $\mathrm{Cu}-\mathrm{Zn}-\mathrm{Al}$ and $\mathrm{Cu}-\mathrm{Al}-\mathrm{Ni}^{3}{ }^{3}$

The martensitic transition is a first-order, diffusionless, solid-solid transition that can be induced by a temperature change or by application of an external stress. The transition is thermoelastic if shape and volume changes (inherent to its first-order nature) are elastically accommodated; the transition is then reversible, with a small associated hysteresis.

The thermodynamic trajectory of the transformation (the evaluation of transformed fraction with temperature and applied stress) depends on an important number of factors such as, for example, the thermomechanical treatments undergone by the specimen, its monocrystalline or polycrystalline character, and the crystallographic structure of the martensite phase. In spite of this complexity, however, the thermodynamic trajectories exhibit a property that is independent of the factors listed and has been repeatedly observed: global memory of the points where transformation direction is reverted (return points). In the present work we focus our attention on this intrinsic property. The situation is comparable to magnetization against magnetic-field trajectories within the global hysteresis loop in ferromagnetic materials. ${ }^{4}$

In particular, we study the evolution of thermodynamic paths inside the complete hysteresis loop of a stressinduced transformation in a $\mathrm{Cu}-\mathrm{Zn}-\mathrm{Al}$ single crystal. In this case all the martensitic plates produced in the transformation have the same orientation. Since investigations have been carried out before on the thermally induced transformation of $\mathrm{Cu}-\mathrm{Zn}-\mathrm{Al}$ single crystals ${ }^{5}$ and polycrystalline $\mathrm{Cu}-\mathrm{Zn}$ (Ref. 6) and $\mathrm{Cu}-\mathrm{Zn}-\mathrm{Al},{ }^{7,8}$ and for the stressinduced transformation of polycrystalline $\mathrm{Cu}-\mathrm{Zn}-\mathrm{Al}$ (Ref. 9) and Ni-Ti. ${ }^{10}$ In all of them the martensitic phase could form in various different orientations, leading to a complex domain structure. In spite of the different domain arrangement, our results show that the memory features of internal paths remain unchanged.

Consequences of the global memory are: (i) the impossibility of using shape-memory alloys as regulators, due to the difficulties in predicting partial stress-strain-temperature trajectories, and (ii) an additional complexity in the thermodynamics of these alloys due to the fact that thermodynamic trajectories are multivalued. ${ }^{5,11}$ In this sense, it is interesting to provide models of the global memory behavior. $\mathrm{Lu}-\mathrm{Li}$ et al. have recently published a thermomechanical model of hysteresis in shape-memory alloys ${ }^{12}$ that reproduces all the features of the memory behavior in a qualitative way; the phenomenological parameters input to the model, however, cannot be obtained explicitly from actuảl measurements, and thus quantitative modeling of a given experimental situation is not possible. To overcome this difficulty, we suggest here the use of Preisach modeling. This model was introduced by Preisach in 1935 (Ref. 13) to describe magnetic hysteresis loops in ferromagnetic materials, and was developed independently for adsorption hysteresis by Everett, Whitton, and Smith more than 15 years later. ${ }^{14-17}$ Several versions of the original (scalar) model have since then appeared regularly in the literature of magnetism, ${ }^{18-25}$ as well as generalizations to two-dimensional and three-dimensional vector models. ${ }^{26-30}$ Among these contributions, Mayergoyz has recently established the mathematical basis of the model ${ }^{31,32}$ and developed a method for its numerical implementation. ${ }^{33}$

The paper is organized as follows: In Sec. II we describe crystal preparation and the experimental procedure. Experimental stress-strain trajectories are presented in Sec. 
III, with emphasis on the partial hysteresis behavior. In Sec. IV we present the Preisach model and adapt it to our problem. We also derive explicit expressions for the trajectories as a function of a limited set of experimental curves (first-order reversal curves), and finally compare calculated and experimental results. Conclusions of the work are presented in Sec. $V$.

\section{EXPERIMENT}

A Cu-Zn-Al single crystal of nominal composition $\mathrm{Cu}$ 19.4 Zn-13.1 Al (at. \%) and nominal transformation temperature $M_{s}=243 \mathrm{~K}$ was chosen for the present experiments. In this composition range the crystal is able to undergo a thermally or stress-induced thermoelastic martensitic transformation, from an ordered $\beta_{1}$ (bcc) phase to a $\beta_{1}^{\prime}(18 R)$ structure. The original ingot was obtained by melting weighted amounts of the initial components ( $99.99 \%$ purity) in an induction furnace. Single crystals were grown from the polycrystalline alloy in an evacuated quartz tube using a modified Bridgman method. The crystals were then carefully machined to a rod tensile shape ( $3.0 \mathrm{~mm}$ diam in the tensile part, $30.5 \mathrm{~mm}$ gauge length).

One of the crystals was then selected and subjected to the following thermal treatment: annealing in the bcc $\beta$ phase at $1023 \mathrm{~K}$ for $45 \mathrm{~min}$, followed by a quench in water at $303 \mathrm{~K}$, to retain the $\beta$ phase as a metastable phase, and aging at this temperature for another $45 \mathrm{~min}$ to ensure completion of $L 2_{1}$ ordering.

The tensile experiments described in the sequel were all performed at room temperature in a strain-controlled INSTRON machine equipped with a 100-kg load cell. Specimen deformation was measured by means of a 12.5$\mathrm{mm}$ gauge length extensometer. Two series of measurements ( $I$ and II) were performed which slightly differ in the position of the extensometer with respect to the tensile gauge length of the crystal, and do not admit mutual comparison because they correspond to two different transformation sequences in slightly different regions of the crystal. The measurements in each series showed separately a reproducibility of the tensile curve better than 0.001 in either stress or strain.

Cross-head speeds from 0.05 to $5.0 \mathrm{~mm} \mathrm{~min}^{-1}$ were used in the experiments, though $0.5 \mathrm{~mm} \mathrm{~min}{ }^{-1}$ was selected as a standard value, used in all the measurements unless otherwise stated. At this strain rate, the experimental accuracy in defining the limits of the tensile test was about $1 \mathrm{MPa}$ in stress and 0.1 in strain.

\section{HYSTERESIS BEHAVIOR IN PARTIAL CYCLING}

Figure 1 shows the stress-strain behavior of the $\mathrm{Cu}-\mathrm{Zn}$ $\mathrm{Al}$ single crystal at room temperature: Large, fully reversible deformations are accessible through a phase transformation mechanism, resulting in a pseudoelastic behavior. ${ }^{1}$ The curve of increasing strain corresponds to a forward thermoelastic martensitic transformation $\beta_{1} \rightarrow \beta_{1}^{\prime}$, and the curve of decreasing strain to the reverse transformation $\beta_{1}^{\prime} \rightarrow \beta_{1}$. The increase in slope at both ends of the two curves is associated with the elastic behavior of the $\beta_{1}$ and $\beta_{1}^{\prime}$

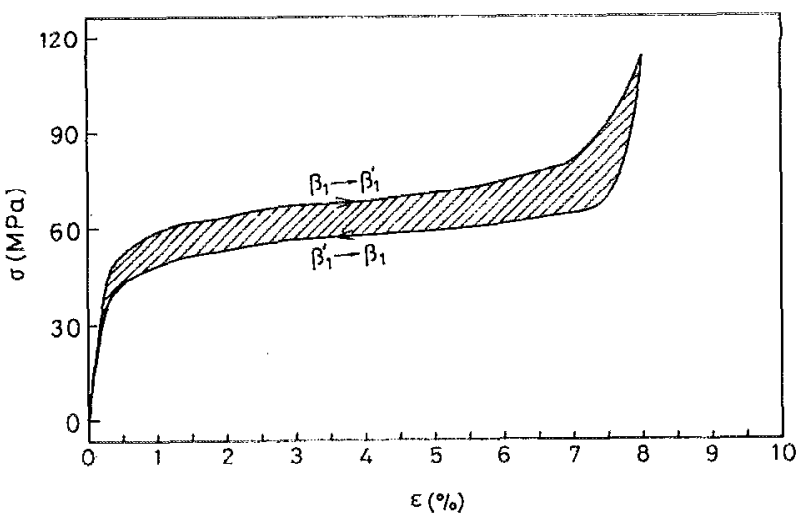

FIG. 1. Pseudoelastic loop followed by a $\mathrm{Cu}-19.4 \mathrm{Zn}-13.1 \mathrm{Al}$ (at. \%) single crystal in a tensile test at room temperature. Cross-head speed is 0.5 $\mathrm{mm} \min ^{-1}$; series I.

phases. Though the specimen deformation is completely recovered at the end of the unloading curve, a hysteresis loop is formed which evidences energy dissipation. Actually the area enclosed within the hysteresis loop (which represents the two-phase region of the alloy at the test temperature) is proportional to the energy lost in the cyclic process. $^{34}$

The shape of the hysteresis loop is sensitive to the transformation rate, as shown in Fig. 2. High strain rates enlarge the hysteresis loop, particularly at the end of both (forward and reverse) transformation paths. This behavior, previously observed by different authors, ${ }^{35,36}$ can be explained by the progressive difficulty in evacuating or absorbing the transformation latent heat at high strain rates. At moderate and low strain rates, however, the hysteresis loop tends asymptotically to be independent of the strain rate, as shown in the figure. Moreover, the reproducibility of a tensile experiment is excellent at a given cross-head speed.

Partial cycling in the two-phase region leads to transformation paths inside the global hysteresis loop, as shown in Fig. 3. In this case the partial cycles simply consist of short unloading-loading excursions from the ascending

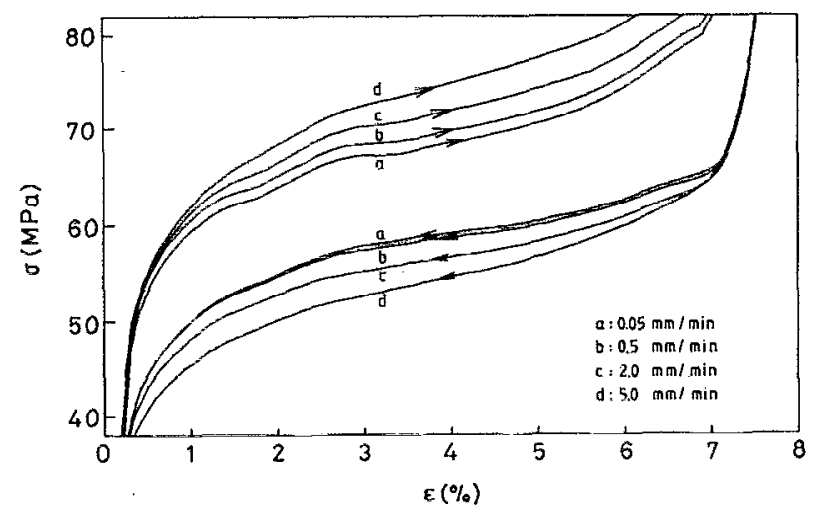

FIG. 2. Influcnce of the cross-head specd on the shape of the complete hysteresis loop; series II. 


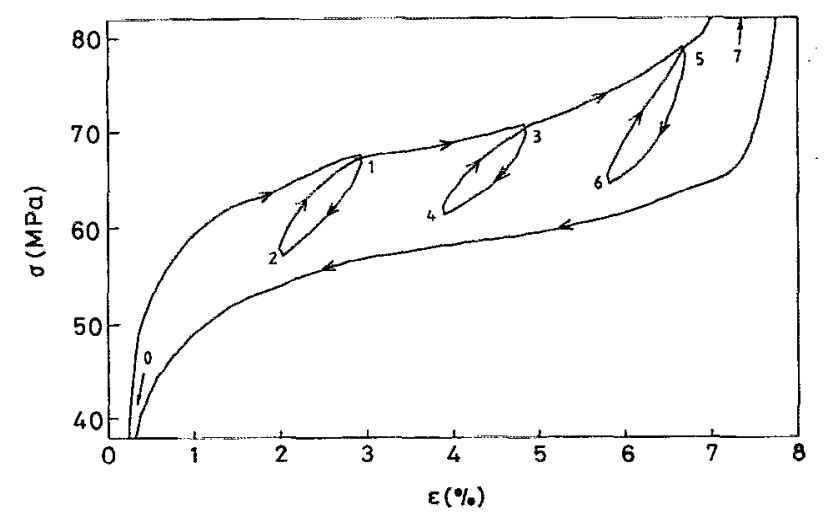

FIG. 3. Partial hysteresis loops obtained by short unloading-loading excursions along the forward transformation path; series II.

branch of the complete loop; consider one of these partial cycles, such as the $1 \rightarrow 2 \rightarrow 1$. It is clear that the transformation path in this inner cycle depends on the loading history: It "remembers" point 1 , at which unloading began, and returns to this point upon subsequent loading $2 \rightarrow 1$. Once this point is crossed, however, the transformation follows the original path, "forgetting" that a partial loop had been performed previously. It is also worth noting that the unloading portion $1 \rightarrow 2$, if continued, would go back to the initial point 0 (see Fig. 9), in the same way as the portion $2 \rightarrow 1$ goes to point 1 . This shows that the complete loop, concerning hysteresis, behaves exactly as an internal partial loop, except for the fact that it represents the boundary of the two-phase region: No transformation paths are allowed beyond this boundary.

Figure 4 shows an equivalent behavior for more internal loops. In this case a first partial loop $2 \rightarrow 7 \rightarrow 2$ is formed by reloading the crystal at point 2 , before complete unloading; from the ascending branch $2 \rightarrow 7$ of this partial cycle (which as before tends to join the global loop at the point where path reversion took place-point 1 , not shown in the figure) two short unloading-loading cycles, $3 \rightarrow 4 \rightarrow 3$ and $5 \rightarrow 6 \rightarrow 5$, have been performed. Consider, for example, the first of them: It depends on the whole sequence of previous

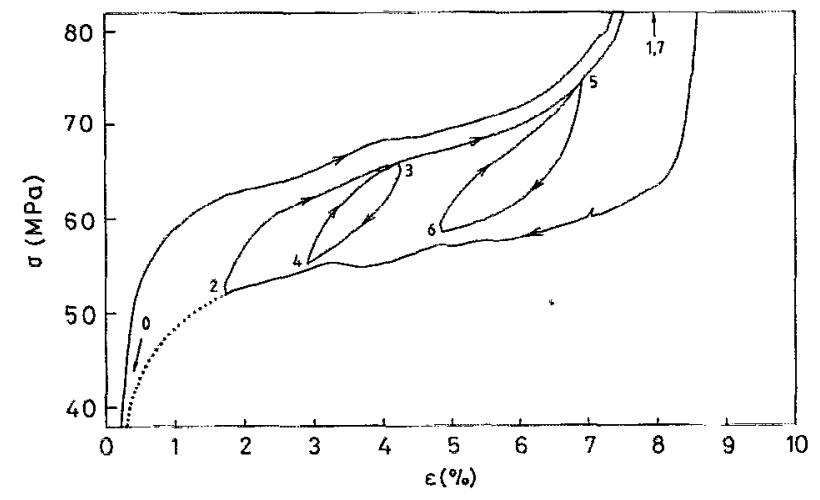

FIG. 4. Partial hysteresis loops $3 \rightarrow 4 \rightarrow 3$ and $5 \rightarrow 6 \rightarrow 5$, performed along a loading path $2 \rightarrow 7$ internal to the two-phase region; series 1 .

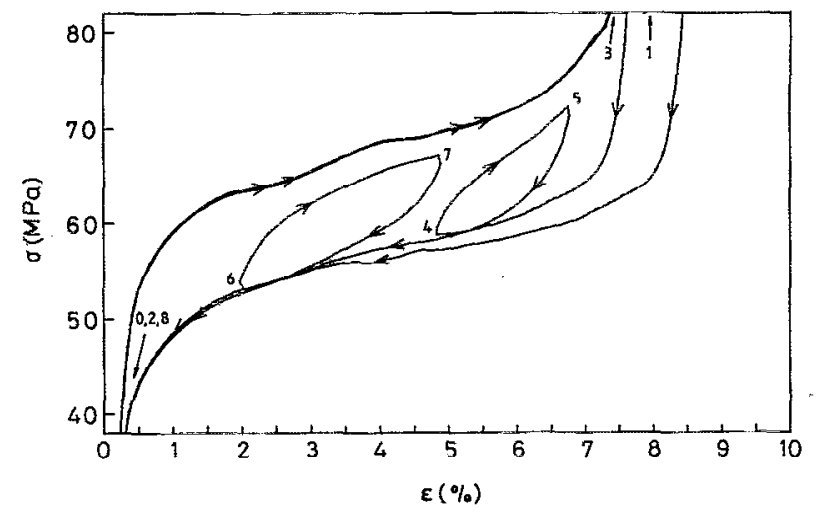

FIG. 5. Partial hysteresis loops $4 \rightarrow 5 \rightarrow 4$ and $6 \rightarrow 7 \rightarrow 6$, performed along an unloading path $3 \rightarrow 8$ internal to the two-phase region; series $I$.

return points; $0,1,2,3$ for the $3 \rightarrow 4$ portion and $0,1,2,3$, 4 for the $4 \rightarrow 3$ portion. This follows from the observation that, for example, the $3 \rightarrow 4$ path, if continued, would go to point 2 , which defined the branch $2 \rightarrow 3$; but the branch $2 \rightarrow 3$, if continued, would have reached point 1 which defined the branch $1 \rightarrow 2$; and finally, this branch $1 \rightarrow 2$, if continued, would have reached point 0 , which on its turn defined the original loading branch $0 \rightarrow 1$. Obviously an equivalent sequence can be traced for the $4 \rightarrow 3$ portion. From the loading/unloading history of the crystal, only the sequence of return points (points of path reversion) have an influence on the future.

The influence of a return point, however, disappears when the point is reached again by the transformation path, closing an internal cycle. This has already been indicated in Fig. 3 and is shown now in Fig. 4 for more internal loops: Path $4 \rightarrow 3$, for example, after reaching point 3 , recovers the original path $2 \rightarrow 7$, losing memory of return points 3 and 4 . By construction, it is clear that this procedure eliminates the influence of the return points in a reverse sequence, the memory of the more recent return points being eliminated first.

Internal loops can also be carried out along an internal unloading branch, corresponding to the $\beta_{1}^{\prime} \rightarrow \beta_{1}$ transformation, as shown in Fig. 5. The internal partial loops $4 \rightarrow 5 \rightarrow 4$ and $6 \rightarrow 7 \rightarrow 6$ reveal again the memorization of the return points 4 and 6 , respectively. There are the following two new features, however, to consider.

(i) The unloading path $5 \rightarrow 4$ does not reach the return point 4 as exactly as we observed for loading paths in Fig. 4 ; instead, it goes a bit below point 4 . The same is true for the $7 \rightarrow 6$ unloading path, but the vicinity of the unloading path $1 \rightarrow 2$ corresponding to the complete hysteresis loop makes this obscrvation difficult.

(ii) The unloading path $7 \rightarrow 8$ goes slightly below the portion $6 \rightarrow 2$ of the unloading path $1 \rightarrow 2$ corresponding to the complete hysteresis loop; this indicates that the memory of reversal point 6 is not perfectly cleared on closing the loop $6 \rightarrow 7 \rightarrow 6$.

Actually, these two features can be summarized in a single observation: A short descending branch tends to go 


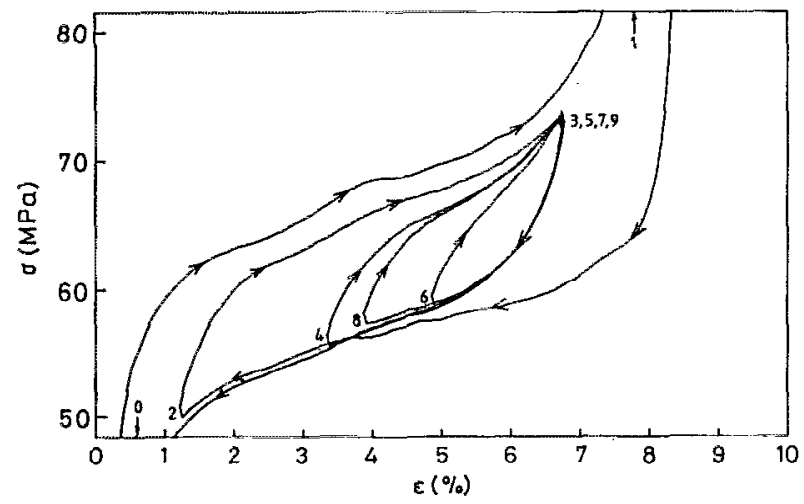

FIG. 6. Internal hysteresis cycles obtained fixing the return point for unloading $(3,5,7,9)$ and performing unloading excursions of varying amplitude; series I.

below a longer one. This is to be associated with the different times available in each case to absorb the latent heat required by the reverse martensitic transformation to proceed in isothermal conditions. Thus, even at cross-head speeds as low as $0.5 \mathrm{~mm} \mathrm{~min}^{-1}$, the reverse transformation paths are still slightly sensitive to the time elapsed from the last return point. We will, nevertheless, neglect this aspect in the modeling of the partial loop behavior described in the following section, and assume a perfectly static hysteresis.

A loop internal to the complete hysteresis loop exhibits the same memory properties as the complete loop. On its turn, a loop internal to this internal loop exhibits again the same memory properties, and so on. Thus the memory properties remain independent of the scale and hierarchy of the loop considered. An example is presented in Fig. 6, which shows a series of internal cycles with a common unloading point $(3,5,7,9)$ and different amplitudes of the unloading excursion. The collection of internal cycles displays equivalent properties to the collection presented in Fig. 9 (collection of "first-order" reversal curves), in spite of having been obtained along the unloading path $3 \rightarrow 4$ already interior to the complete loop. Observing in detail the trajectories in the figure, it comes out that a practically perfect reproducibility of the return point (difficult to achieve in a tensile experiment of this kind) is essential to maintain a good reproducibility of the transformation paths inside the two-phase region. The smallest difference in a turning point between two identical trajectories makes the two trajectories differ noticeably after a few path reversions, even if the latter are exactly reproduced; the reason must be found in the memory properties of the transformation trajectories. This extreme sensitivity to the cycling limits has been previously evidenced by acoustic emission measurements for the thermally induced transformation of monocrystalline $\mathrm{Cu}-\mathrm{Zn}-\mathrm{Al} .^{37}$

As mentioned in Sec. I, the phenomenological characteristics of partial cycling behavior deriving from the ensemble of our observations are totally consistent with the characteristics reported previously for single crystals in

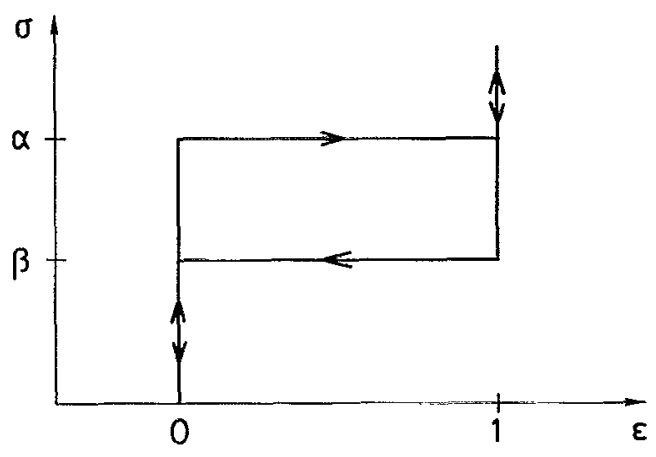

FIG. 7. Definition of an elementary hysteresis operator $\gamma_{\alpha \beta}$.

thermal cycling and for polycrystals in either thermal or stress cycling. In the present isothermal experiments they can be summarized by the following.

(i) All transformation paths are restricted to a region of the $\sigma-\epsilon$ space-the two-phase region. The path resulting from complete forward transformation and complete reverse transformation sets the boundaries of the two-phase region.

(ii) The transformation path followed by the system in the two-phase region depends on its previous history through the ensemble of return points in the path.

(iii) The influence of a return point on the evolution of a transformation path disappears when the transformation path (closing a partial cycle) reaches the return point again.

(iv) If the whole of the transformation path is performed at identical conditions of cross-head speed and temperature, the hysteresis behavior is static: Time is just a parameter in the $\sigma(t)-\epsilon(t)$ relation, and the memory of the return points does not evolve with time.

Mayergoyz ${ }^{32}$ has shown that a hysteretic behavior displaying the above characteristic features, together with a condition of congruency between internal loops of equal extreme $\sigma$ values, is well suited to be represented by a Preisach model. The following section is devoted to a short revision of this model and its application to the experiments described here.

\section{APPLICATION OF THE PREISACH MODEL}

\section{A. Preisach model for hysteresis}

We take Mayergoyz's formulation ${ }^{31}$ of the Preisach model and adapt it to the problem of a stress-induced phase transformation. The control parameter (intensive variable) is the applied uniaxial stress $\sigma$ and the output variable (extensive variable) the resulting deformation $\epsilon$. An important difference with the magnetic case is that $\sigma-\epsilon$ hysteresis loops are only defined in the first quadrant of the $\sigma-\epsilon$ axes. The Preisach elementary hysteresis operator $\gamma_{\alpha \beta}$ must then be defined as in Fig. 7. A superposition of elementary operators with different $\alpha, \beta$ values $(\alpha \geqslant \beta)$ provides the strain $\epsilon(t)$ corresponding to a value $\sigma(t)$ of the applied stress: 

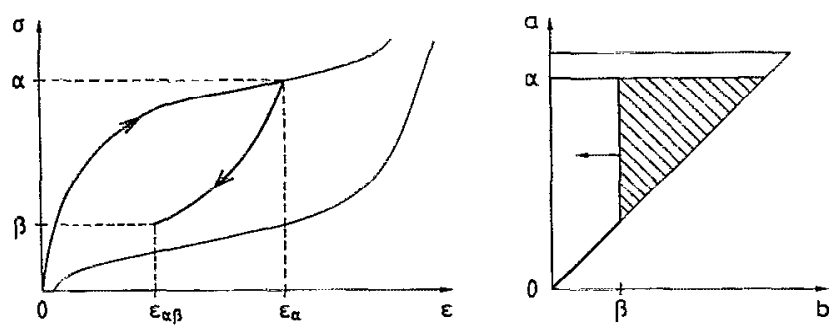

FIG. 8. Left-hand side: determination of $\epsilon_{\alpha}$ and $\epsilon_{\alpha \beta}$ from a first-order reversal trajectory (schematic). Right-hand side: triangular support of integration (shaded) associated with $F(\alpha, \beta)$.

$$
\epsilon(t)=\iint_{\alpha>\beta} \mu(\alpha, \beta)\left[\gamma_{\alpha \beta} \sigma(t)\right] d \alpha d \beta .
$$

Equation (1) is the Preisach model for hysteresis. The function $\mu(\alpha, \beta)$ represents the population of elementary operators with switching values $\alpha, \beta$.

It must be noted that, according to Fig. 7, $\left[\gamma_{\alpha \beta} \sigma(t)\right]$ can only take the values 0 or 1 . Thus, Eq. (1) reduces to

$$
\epsilon(t)=\iint_{S^{+}(t)} \mu(\alpha, \beta) d \alpha d \beta,
$$

where $S^{+}(t)$ is a region (in the triangular support $\alpha \geqslant \beta$ ) containing all the $(\alpha, \beta)$ points of elementary operators in the +1 state at time $t$, and only those points.

\section{B. Evaluation of $\mu(\alpha, \beta)$}

The Preisach model is particularly attractive because the population $\mu(\alpha, \beta)$ of elementary operators can be evaluated from a limited set of experimental data. To this purpose, consider a first-order reversal path, i.e., a path obtained from the main loading branch on unloading at a given $\sigma$ value. Figure 8 shows this kind of path and how to determine the strain values $\epsilon_{\alpha}$ and $\epsilon_{\alpha \beta}$, which in terms of the Preisach model read

$$
\begin{aligned}
\epsilon_{\alpha}= & \iint_{\alpha>a>b>0} \mu(a, b) d a d b, \\
\epsilon_{\alpha \beta}= & \iint_{\alpha>a>b>0} \mu(a, b) d a d b \\
& -\iint_{\alpha>a>b>\beta} \mu(a, b) d a d b .
\end{aligned}
$$

From these two values we can construct the function

$$
F(\alpha, \beta) \equiv \epsilon_{\alpha}-\epsilon_{\alpha \beta},
$$

which, from Eqs. (3) and (4), amounts to

$$
\begin{aligned}
F(\alpha, \beta) & =\iint_{\alpha>a>b>\beta} \mu(a, b) d a d b \\
& =\int_{\beta}^{\alpha} d a \int_{\beta}^{a} d b \mu(a, b) .
\end{aligned}
$$

It is then easy to show that

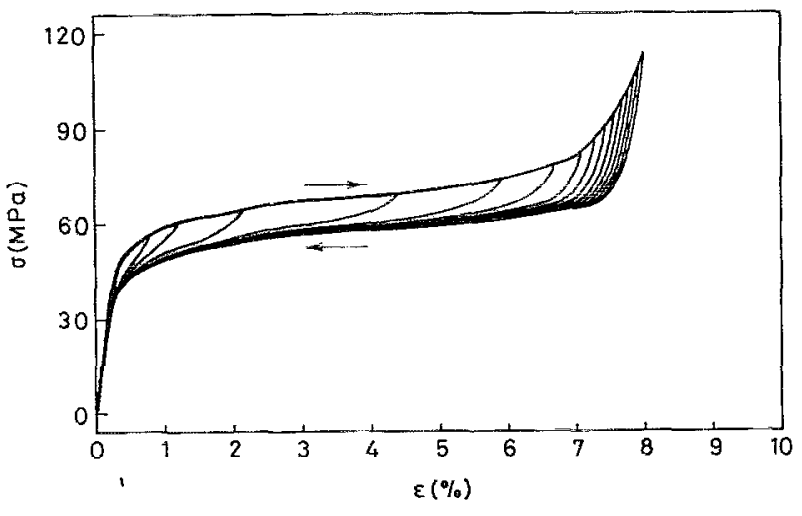

FIG. 9. Collection of first-order reversal curves used to evaluate the function $F(\alpha, \beta)$ in series-I experiments.

$$
\mu(\alpha, \beta)=-\frac{\partial^{2} F(\alpha, \beta)}{\partial \alpha \partial \beta} .
$$

Hence, the population of elementary operators can be calculated from $F(\alpha, \beta)$, which on its turn can be obtained from a collection of experimental first-order reversal paths. This makes the Preisach model particularly attractive: It does not only reproduce the global memory features of partial hysteresis loops, but it is able to predict as well the trajectories using a limited set of experimental data.

Part of the collection of experimental first-order reversal curves used to compute $F(\alpha, \beta)$ in series-I experiments is plotted in Fig. 9. Values of $F(\alpha, \beta)$ on an evenly spaced grid of $33 \alpha$ and $196 \beta$ values have been obtained from numeric interpolation between these curves; a bicubic spline interpolation algorithm ${ }^{38}$ on the evenly spaced grid, finally, has enabled computation of $F(\alpha, \beta)$ at any point $(\alpha, \beta)$ within the grid.

\section{Computation of trajectorles Inside the two-phase region}

Starting from a well-defined origin, trajectories predicted by the Preisach model inside the two-phase region can be computed using Eq. (2). This equation involves a double integral of $\mu(\alpha, \beta)$, which in turn is obtained as the second derivative of $F(\alpha, \beta)$, as Eq. (7) shows. It follows then that, given an evolution of the input $\sigma(t)$, explicit expressions for the output $\epsilon(t)$. can be obtained that involve only $F(\alpha, \beta)$ and not $\mu(\alpha, \beta)$ (Ref. 33); this procedure avoids the important uncertainties associated with a double numeric derivative of the function $F(\alpha, \beta)$, affected by experimental noise.

The purpose of this subsection is to derive explicit expressions of $\epsilon(t)$, which depend only on $F(\alpha, \beta)$, for the two general types of trajectories considered here: ascending $A$ and descending $D$ branches. The starting point of all trajectories is considered to be the point $\sigma=0, \epsilon=0$ where the crystal is in the parent phase with no stress applied. The derivation illustrates as well how the model keeps memory of the input extrema. It is based on the observa- 

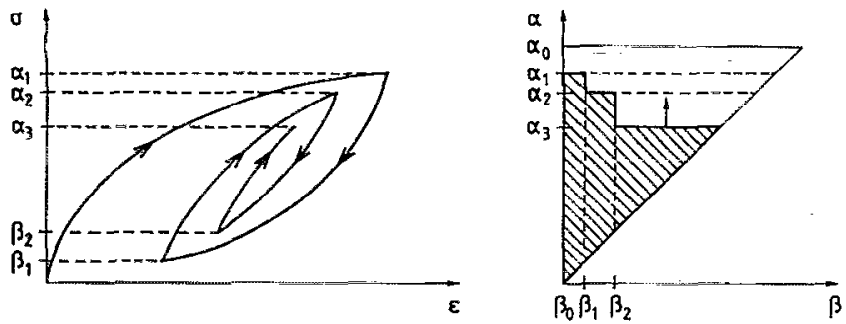

FIG. 10. Schematic representation of an ascending branch $A$ and its corresponding staircase line $S^{+}$defining the support of integration of $\mu(\alpha, \beta)$ (shaded).

tion that, according to Eq. $(6), F(\alpha, \beta)$ is the double integral of $\mu(\alpha, \beta)$ on the triangular support drawn shaded in Fig. 8.

For an $A$ branch following from a number of reversions (see Fig. 10) $\epsilon(t)$ is the double integral of $\mu(\alpha, \beta)$ on a support defined by a staircase line $S^{+}(t)$ whose last segment is horizontal. Hence, constructing this support as a superposition of triangular supports,

$$
\begin{aligned}
\epsilon(t)= & \iint_{S^{+}(t)} \mu(\alpha, \beta) d \alpha d \beta \\
= & {\left[F\left(\alpha_{1}, \beta_{0}\right)-F\left(\alpha_{1}, \beta_{1}\right)\right]+\left[F\left(\alpha_{2}, \beta_{1}\right)\right.} \\
& \left.-F\left(\alpha_{2}, \beta_{2}\right)\right]+F\left(\alpha_{3}, \beta_{2}\right)
\end{aligned}
$$

or, in general,

$\epsilon(t)=F\left(\alpha_{0}, \beta_{0}\right)-\sum_{i=0}^{N}\left[F\left(\alpha_{i}, \beta_{i}\right)-F\left(\alpha_{i+1}, \beta_{i}\right)\right]$.

For a $D$ branch following as well from a number of reversions, $\epsilon(t)$ is the double integral of $\mu(\alpha, \beta)$ on a support defined by a staircase line $S^{+}(t)$ whose last segment is vertical (Fig. 11). In this case,

$$
\begin{aligned}
\epsilon(t)= & \iint_{S^{+}(t)} \mu(\alpha, \beta) d \alpha d \beta \\
= & {\left[F\left(\alpha_{1}, \beta_{0}\right)-F\left(\alpha_{1}, \beta_{1}\right)\right]+\left[F\left(\alpha_{2}, \beta_{1}\right)\right.} \\
& \left.-F\left(\alpha_{2}, \beta_{2}\right)\right]+\left[F\left(\alpha_{3}, \beta_{2}\right)-F\left(\alpha_{3}, \beta_{3}\right)\right]
\end{aligned}
$$

or, in general,
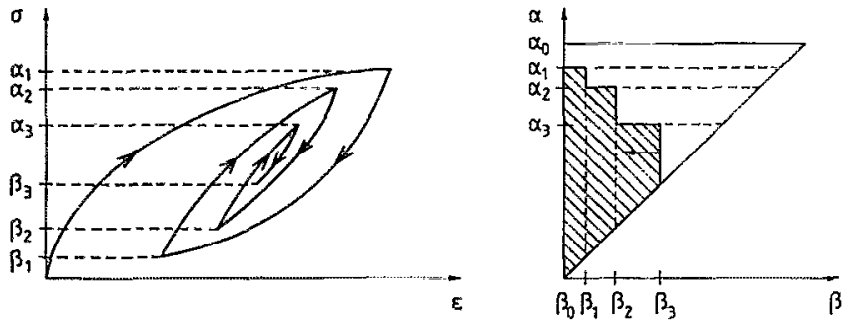

FIG. 11. Schematic representation of a descending branch $D$ and its corresponding staircase line $S^{+}$defining the support of integration of $\mu(\alpha, \beta)$ (shaded).

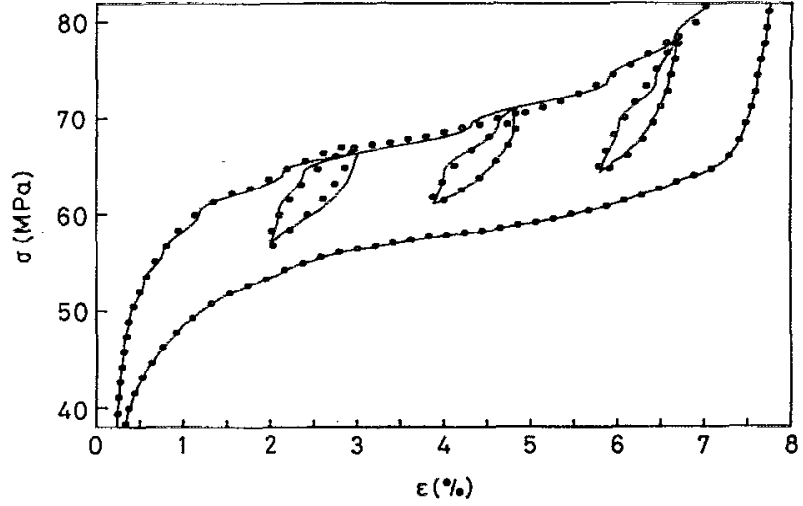

FIG. 12. Comparison between experimental ( $)$ and calculated (solid line) trajectories; series II.

$$
\epsilon(t)=\sum_{i=0}^{N-1}\left[F\left(\alpha_{i+1}, \beta_{i}\right)-F\left(\alpha_{i+1}, \beta_{i+1}\right)\right]
$$

\section{Comparison to experimental results}

We have used Eqs. (5), (9), and (11) to simulate some of the experimental trajectories available.

Figure 12 presents a first comparison between experimental (black dots) and calculated (solid line) trajectories. The agreement is excellent in this case, and shows already the ability of the model to retain and to erase memory of the return points. Small fluctuations in the calculated trajectories arise from the interpolation algorithm.

Figure 13 is another example of the trajectories predicted by the model, presenting in this case partial loops internal to other partial loops. It must be emphasized that, while descending branches are accurately reproduced, departures of the calculated ascending branches from the experimental ones are significant. Though this dissymmetry in the accuracy of the model could be partially due to the significant difference in density of $\alpha$ and $\beta$ values in the $(\alpha, \beta)$ grid, it is also an indication that a qualitatively different hysteretic behavior is displayed by $\beta_{1} \rightarrow \beta_{1}^{\prime}$ and $\beta_{1}^{\prime} \rightarrow \beta_{1}$ transformations (corresponding to $A$ and $D$

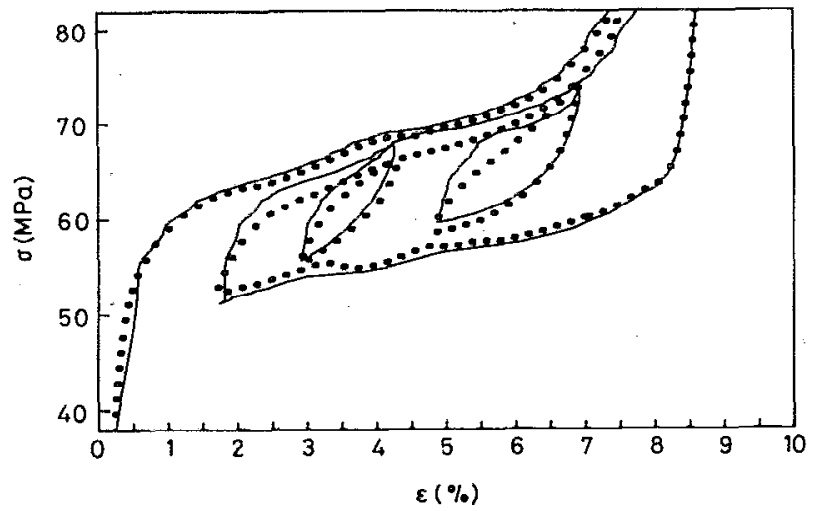

FIG. 13. Comparison between experimental (๑) and calculated (solid line) trajectories, including internal hysteresis loops; series I. 


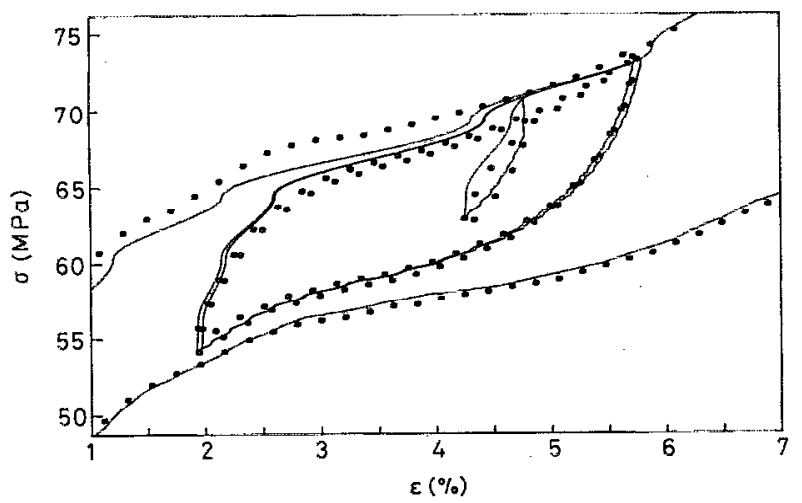

FIG. 14. Comparison between experimental (๑) and calculated (solid line) trajectories; series I.

branches of the $\sigma-\epsilon$ trajectories, respectively). A possible difference in the dissipative mechanisms operative in forward and reverse transformation is also consistent with the different amount of acoustic emission systematically observed in the two directions of transformation. ${ }^{39-41}$ It turns out that in our calculation $D$ branches are accurately reproduced because the set of first-order reversal curves used to evaluatc $F(\alpha, \beta)$ is itself a set of $D$ branches.

A final example of calculated trajectories is presented in Fig. 14, showing also a better reproducibility of $D$ branches compared to $A$ branches. The fact that an internal trajectory depends (through the memory of return points) on all the previous external trajectories makes that a slight inaccuracy in reproducing the external curves propagates to produce an uncontrolled degree of inaccuracy in the internal curve.

\section{CONCLUSIONS}

The stress-strain trajectories followed by a $\mathrm{Cu}-\mathrm{Zn}-\mathrm{Al}$ single crystal under uniaxial tensile solicitation have been investigated. Measurements have been performed at room temperature, where the alloy displays pseudoelasticity through a thermoelastic martensitic transformation. Particular attention has been paid to the properties of partial hysteresis loops in the two-phase region.

Our results are coincident with all the results reported in the literature for stress-induced transformations in polycrystals and thermally induced transformations in single crystals and polycrystals, in spite of the fact that in the prcsent experiments the martensitic phase forms in only one orientation, out of the 24 equivalent ones derived from the crystallographic symmetry of the $\beta_{1}$ bcc phase, and the domain arrangement in the two-phase region consists therefore on a simple alternate sequence of single-oriented $\beta_{1}$ and martensitic domains. This proves that the characteristic sub-loop behavior in the martensitic transformation is not inherently related to the presence of differently oriented martensitic domains.

Essential features of the partial loop behavior that have been found are: (i) The transformation trajectories inside the two-phase region depend on the sequence of previous inversion points, which the system happens to memorize, and (ii) the memory of a previous inversion point is erased when the trajectory runs over the given point again.

This behavior, in which time plays no role other than a parameter and hysteresis is therefore static, is actually an idealization that the system tends to follow at very low transformation rates. Actually, the latent heat of transformation is exchanged between specimen and ambience at limited velocity, and difficulties in transferring the heat at higher or the same rate at which transformation is driven results in additional (extrinsic) hysteresis, which is not static anymore but distorts differently trajectories of different duration. The situation can be viewed from another side: The $\sigma-\epsilon$ trajectories are no longer isothermal and the hysteresis behavior should then be studied in a $\sigma-\epsilon-T$ space, where $T$ refers to the specimen temperature.

A version of the Preisach model has been formulated to predict the partial cycling behavior of the crystal from a collection of experimental first-order reversal curves. This collection suffices to determine the function $F(\alpha, \beta)$ and, from it, explicit expressions of any trajectories in the twophase region, with no adjustable parameters.

Our calculated trajectories reproduce the two essential features of the partial loop behavior listed above, and are able to predict the experimental trajectories quantitatively; the prediction is precise for $D$ branches $\left(\beta_{1}^{\prime} \rightarrow \beta_{1}\right.$ transformation), while calculated $A$ branches $\left(\beta_{1} \rightarrow \beta_{1}^{\prime}\right.$ transformation) systematically follow higher values of stress than the corresponding experimental ones. This reveals a dissymmetry between forward and reverse martensitic transformation concerning the accompanying dissipative processes. A dissymmetry is also observed in measurements of acoustic emission in the transformation, and points out a need of extending the Preisach model in a way that incorporates experimental information from first-order reversal curves corresponding to both $A$ and $D$ branches.

\section{ACKNOWLEDGMENTS}

The experimental support received from the Department of Metallurgy and Materials Engineering of the Katholieke Universiteit Leuven, in Belgium, is gratefully acknowledged. The author is also grateful to Professor Luc Delaey for introducing him to the subject and for his encouragement. This research is partially supported by DGICyT (Spain), Project No. MAT 89-0748, and the publication charges by CIRIT (Generalitat de Catalunya).

${ }^{1}$ L. Delaey, R. V. Krishnan, H. Tas, and H. Warlimont, J. Mater. Sci. 9, 1521 (1974).

${ }^{2}$ C. M. Wayman, in Proceedings of the ICOMA T"86 (The Japan Institute of Metals, Nara, 1987), pp. 645-652.

${ }^{3}$ H. Warlimont and L. Delaey, Progr. Mater. Sci. 18, 1 (1974).

${ }^{4}$ D. L. Jiles and D. L. Atherton, J. Appl. Phys. 55, 2115 (1984).

${ }^{5}$ A. Planes, T. Castán, J. Ortín, and L. Delaey, J. Appl. Phys. 66, 2342 (1989).

${ }^{6}$ I. Cornelis and C. M. Wayman, Scr. Metall. 10, 359 (1976).

${ }^{7}$ W. Zhongguo and Y. Dazhi, Scr. Metall. 22,1245 (1988).

${ }^{8}$ Y. I. Paskal and L. A. Monasevich, Phys. Met. Metall. 52, 95 (1981).

${ }^{9}$ L. Delaey, J. Van Humbeeck, M. Chandrasekaran, J. Janssen, M. Andrade, and N. Mwamba, Met. Forum 4, 164 (1981).

${ }^{10}$ J. L. McNichols, Jr. and J. S. Cory, J. Appl. Phys. 61, 972 (1987). 
1'J. S. Cory and J. L. McNichols, Jr., J. Appl. Phys. 58, 3282 (1985).

${ }^{12} \mathrm{Lu} \mathrm{Li}$, E. Aernoudt, P. Wollants, J. Yan Humbeeck, and L. Delaey, Z. Metallkd. 81, 613 (1990).

${ }^{13}$ F. Preisach, Z. Phys. 94, 277 (1935).

${ }^{14}$ D. H. Everett and W. I. Whitton, Trans. Faraday Soc. 48, 749 (1952).

${ }^{15}$ D. H. Everett and F. W. Smith, Trans. Faraday Soc. 50, 187 (1954).

${ }^{16}$ D. H. Everett, Trans. Faraday Soc. 50, 1077 (1954).

${ }^{17}$ D. H. Everett, Trans. Faraday Soc. 51, 1551 (1955).

${ }^{18} \mathrm{G}$. Biorci and D. Pescetti, Nuovo Cimento 7, 829 (1958).

${ }^{19}$ J. A. Barker, D. E. Schreiber, B. G. Huth, and D. H. Everett, Proc. R. Soc. London Ser. A 386, 251 (1983).

${ }^{20}$ I. D. Mayergoyz and G. Friedman, J. Appl. Phys. 61, 3910 (1987).

${ }^{21}$ G. Kádár and E. Della Torre, IEEE Trans. Magn. MAG-23, 2820 (1987).

${ }^{22}$ E. Della Torre and G. Kádár, IEEE Trans. Magn. MAG-23, 2823 (1987).

${ }^{23}$ G. Kádár, J. Appl. Phys. 61, 4013 (1987).

${ }^{24}$ G. Kádár and E. Della Torre, J. Appl. Phys. 63, 3001 (1988).

${ }^{25}$ M. Brokate, IEEE Trans. Magn. MAG-25, 2922 (1989).

${ }^{26} \mathrm{E}$. Della Torre, J. Appl. Phys. 61, 4016 (1987).
${ }^{27}$ K. Wiesen and S. H. Charap, J. Appl. Phys. 61, 4019 (1987).

${ }^{28}$ I. D. Mayergoyz and G. Friedman, J. Appl. Phys. 61, 4022 (1987).

${ }^{29}$ I. D. Mayergoyz, J. Appl. Phys. 63, 2995 (1988).

${ }^{30}$ E. Della Torre and G. Kádár, J. Appl. Phys. 63, 3004 (1988).

${ }^{31}$ I. D. Mayergoyz, J. Appl. Phys. 57, 3803 (1985).

${ }^{32}$ I. D. Mayergoyz, Phys. Rev. Lett. 56, 1518 (1986).

${ }^{33}$ T. Doong and I. D. Mayergoyz, IEEE Trans. Magn. MAG-21, 1853 (1985).

${ }^{34}$ J. Ortín and A. Planes, Acta Metall. 37, 1433 (1989).

${ }^{35}$ J. Van Humbeeck and L. Delaey, J. Phys. (Paris) Colloq. 42, C5-1007 (1981).

${ }^{36}$ C. Rodriguez and L. C. Brown, Met. Trans. A 11, 147 (1980).

${ }^{37}$ F. C. Lovey, J. Ortin, and V. Torra, Phys. Lett. A 121, 351 (1987).

${ }^{38}$ W. H. Press, B. P. Flannery, S. A. Teukolsky, and W. T. Vetterling, Numerical Recipes (Cambridge University Press, Cambridge, 1986), pp. 98-100.

${ }^{39}$ I. Y. Ievlev, V. P. Melekhin, R. I. Mints, and V. M. Segal, Sov. Phys. Solid State 15, 1761 (1974).

${ }^{40}$ J. Baram and M. Rosen, Acta Metall. 30, 655 (1982).

${ }^{41}$ Ll. Mañosa, A. Planes, and E. Cesari, J. Phys. D 22, 977 (1989). 